\title{
Computational Technology for Global Search Based on the Modified Algorithm of the Univariate Nonlocal Optimization
}

\author{
Alexander Gornov \\ Matrosov Institute for System \\ Dynamics and Control Theory of \\ Siberian Branch of the Russian \\ Academy of Sciences \\ Irkutsk, Russia \\ gornov@icc.ru
}

\author{
Pavel Sorokovikov \\ Matrosov Institute for System \\ Dynamics and Control Theory of \\ Siberian Branch of the Russian \\ Academy of Sciences \\ Irkutsk, Russia \\ pavel2301s@gmail.com
}

\author{
Tatiana Zarodnyuk \\ Matrosov Institute for System \\ Dynamics and Control Theory of \\ Siberian Branch of the Russian \\ Academy of Sciences \\ Irkutsk, Russia \\ tz@icc.ru
}

\begin{abstract}
The paper presents an approach to the numerical study of the problems of finding a global extremum of multiextremal functions, based on the use of a parabolas algorithm. As local methods of onedimensional search, the methods of parabolic interpolation and the golden section are used. The numerical testing of modifications of the implemented approach using known non-convex functions has been carried out. The proposed technique has been applied to investigate a more complex optimization problem of a controlled dynamical power system. The obtained numerical results allowed us to demonstrate the efficiency of the proposed computational technology.
\end{abstract}

Keywords-global optimization problem, parabolas algorithm, computational technology, power system.

\section{INTRODUCTION}

The problems of a global extremum search continue to be topical issues arising in various areas of human activity. The emergence of new problems leads to the need to develop new methods for their research. At present, approaches in this area are well developed, based on estimates of the Lipschitz constant of optimized functions [1], and the use of interval analysis methods [2]. For effective application of these methods, it is necessary to have good estimates of the algorithmic parameters and, as a rule, are limited in the dimensions of the studied problems. Methods are also being developed for successively finding local extremes (for example, tunnel search), including those based on the multistart ideology [3]. Here, as in most methods of global search, there is the problem of increasing the guarantees of finding a global extremum. This paper proposes an approach that uses the advantages of a multistart, specifically covering an admissible region with a starting grid, local search capabilities that allow us to achieve extreme values with high accuracy and also the idea of choosing promising areas of search, allowing to save computing resources and increase the likelihood of achieving the extremum. The proposed technique has been applied to investigate a more complex optimization problem of the controlled dynamical power system.

\section{THE CONSIDERABLE GLOBAL OPTIMIZATION PROBLEM}

The paper considers the box-constrained problem of global optimization:

$$
\begin{aligned}
& f(x) \rightarrow \min , x \in B, \\
& B=\left\{x \mid x=\left(x_{1}, x_{2}, \ldots, x_{n}\right), \alpha_{i} \leq x_{i} \leq \beta_{i}\right\},
\end{aligned}
$$

where $f(x)$ is the non-convex and smooth objective function, $n$ is the dimension of the vector $x$ from the intervals $\alpha_{i} \leq x_{i} \leq \beta$.

The presented problem can be helpful in the study of optimal control problems in the following statement:

$$
\frac{d x}{d t}=f(t, x, u), t \in\left[t_{0}, T\right], x(t) \in \Omega \subset R^{n},
$$

where $u=u(t)$ is the control from a class of piecewise continuous vector functions with values in a set $U: u(t) \in U \subset R^{k}$. We assume that the domain of admissible states of the system $\Omega$ and the function $f(t, x, u)$ are such that the solution of the Cauchy problem exists and is unique.

It is necessary to minimize the objective functional

$$
I_{0}(u)=\varphi_{0}(t, x, u) \rightarrow \min .
$$

This optimization of the controlled dynamical system can be considered as the search for the extremal value of the aim function on a reachable set of the corresponding system:

$$
x^{*}: \varphi_{0}(x) \rightarrow \min , x \in D,
$$

where the point $x^{*}$ is the point at which is achieved the minimal value of the function. The reachable set is defined in the following way

$$
D=\left\{x\left(t_{1}\right) \in R^{r}: \dot{x}=f(x(t), u(t), t), x\left(t_{0}\right)=x^{0},\right.
$$




$$
u(t) \in U, t \in T\} .
$$

To investigate this problem, we can apply, among others, the approaches developed in the theory of mathematical programming, for example, gradient type methods, covering methods and etc. For this, the function must be continuous along with its derivative. The difficulties of solving the formulated problem arise from the possible nonconvexity of both the objective function and the admissible set. We propose the "parabolas" method for study the auxiliary problem of one-dimensional optimization which allows us to search the global extremum.

\section{COMPUTATIONAL TECHNOLOGY FOR NON-LOCAL EXTREMUM SEARCH}

Any method of searching for a global extremum includes two stages: a scanning search for an extremum on the whole admissible set and an exact local search in those areas where the global extremum is most likely. The proposed approach allows the design of computational schemes that fully satisfy these requirements, and be the basis of effective algorithms for solving global optimization problems.

\section{A. The univariate parabolas algorithm}

The parabolas method of nonlocal one-dimensional minimization is based on the search for parabolic minima formed by "convex triples" [4-5]. It was successfully used as an auxiliary algorithm in a number of applications, including for solving optimal control problems [6]. A modification of the parabolas method with the selection of "convex triples" was proposed. In this algorithm, the search for minima is not all parabolas, but only the "best" (by value at the midpoint).

The univariate algorithm:

Step 1. On the interval $[\alpha, \beta]$ of changing values of a variable $x$, a grid $C=\left\{c_{i}\right\}_{i=\overline{1, N}}$ is randomly generated, such that $c_{1}=\alpha, c_{N}=\beta$, where $N$ is the number of samples (algorithm parameter).

Step 2. The values of the function $f\left(c_{i}\right), i=\overline{1, N}$ are calculated.

Step 3. Each successively placed triple of points $c_{i-1}<c_{i}<c_{i+1}$ is checked for "convexity": $f\left(c_{i-1}\right) \geq f\left(c_{i}\right) \leq f\left(c_{i+1}\right)$. If the inequality holds, then the triple of points $\left\langle c_{i-1}, c_{i}, c_{i+1}\right\rangle$ is "convex".

Step 4. Found "convex triples" $\left\langle c_{i-1}, c_{i}, c_{i+1}\right\rangle$ are sorted by the value $f\left(c_{i}\right)$.

Step 5. "Convex triples" with the smallest values $f\left(c_{i}\right)$ are selected ( $K \%$ of the total number of "convex triples", where $K$ is the parameter of the algorithm).
Step 6. For each selected "convex triple", the function is minimized $\min _{x \in\left[c_{i-1}, c_{i+1}\right]} f(x)$ using a combination of local one-dimensional search methods.

Step 7. Among the values $f(x)$ found in each "convex triple", the smallest one on the interval $[\alpha, \beta]$ is selected.

Step 8 . The resulting point $x^{*}$ is the solution.

As local methods of one-dimensional search, the methods of parabolic interpolation and the golden section are used.

\section{B. The multivariate scheme}

The approach to solving the considered finitedimensional problem (1)-(2) is based on a combination of the following methods: coordinate descent for the multidimensional problem of finding the extremum and parabolas method for auxiliary nonlocal onedimensional search (see Section 4.1). Let us describe one iteration of the multivariate scheme for solving the problem (1) - (2).

At the $k$-th step of the multivariate scheme:

1. All coordinates of the point $x\left(x_{1}, x_{2}, \ldots, x_{k}, \ldots, x_{n}\right)$ are fixed, except $x_{k}$, where:

1.1. $k=\overline{1, n}$, is selected sequentially (the variants $01,02)$, or

1.2. $k=\operatorname{rand}(1, n)$, is randomly selected, where rand $(1, n)$ is the function to generate an integer in the range from 1 to $\mathrm{n}$ (the variants 03, 04).

2. The one-dimensional function $f\left(x_{k}\right)$ is minimized by the parabolas method (see Section 4.1), where:

2.1. All convex triples are considered, $K=100 \%$ (the variants 01,03), or

2.2. Half of all convex triples are considered, $K=50 \%$ (the variants 02,04 ).

Iteration is complete.

\section{NUMERICAL EXAMPLES}

The developed computational technology was implemented in $C$ language using uniform software standards. To study the numerical properties of the proposed technology, a series of computational experiments has been carried out. Calculations were performed using a server computer with the following characteristics: 2 x Intel Xeon E5-2680 v2 $2.8 \mathrm{GHz}(20$ cores, 40 threads); 128 Gb DDR3 $1866 \mathrm{MHz}$.

\section{A. Testing of the univariate algorithm}

To demonstrate the idea of the proposed parabolas algorithm, it is convenient to use a non-linear onedimensional function. As an example, consider the 
objective function $f(x)=(3 x-1.4) \sin (18 x)$, $x \in[0,1.2]$ (fig 1 ).

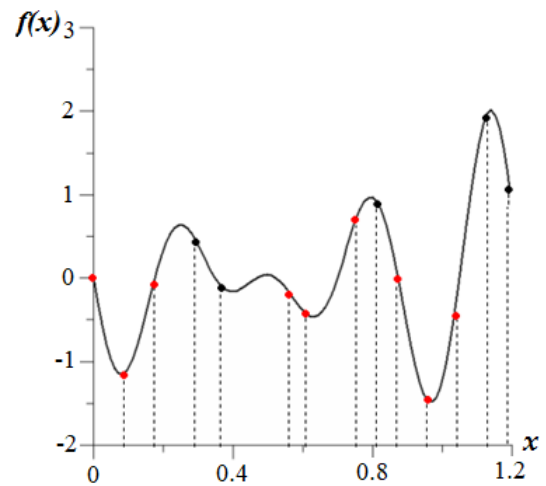

Fig. 1. A set of points containing convex triples for non-linear onedimensional function

This set of points contains three convex triples. The global extremum is in the extreme right triple. To find it, it is enough to run a local minimization algorithm in each of the triples, and remove the remaining areas from consideration. The obtained minimal value is equal 1.48907 and achieved by $1 \mathrm{sec}$. of CPU time.

\section{B. Testing of the multivariate scheme}

A test of the developed computational technology was performed. Various non-convex test functions were considered as test problems. The dimension of the problems is 10 variables. A comparison of four variants of a multidimensional scheme has been carried out, which differ from each other either by the coordinate selection method or by the parameter K value (see Table 1). For each variant, the $\delta=\left|\tilde{f}-f^{*}\right|$ value was calculated, where $\tilde{f}$ is the value found by the algorithm, $f^{*}$ is the global minimum value.

TABLE I. VARIANTS OF THE MULTIDIMENSIONAL SCHEME

\begin{tabular}{ccc}
\hline Variant & $\begin{array}{c}\text { Coordinate selection } \\
\text { method }\end{array}$ & $\begin{array}{c}\text { Value } \\
\text { of K }\end{array}$ \\
\hline 1 & $k=\overline{1, n}$ & $100 \%$ \\
2 & $k=\overline{1, n}$ & $50 \%$ \\
3 & $k=\operatorname{rand}(1, n)$ & $100 \%$ \\
4 & $k=\operatorname{rand}(1, n)$ & $50 \%$
\end{tabular}

1) Rosenbrock function

The Rosenbrock function is a non-convex function used for the numerical study of optimization algorithms [7] proposed by Howard Rosenbrock in 1960 [8]. Finding a global minimum for this function is a nontrivial problem due to its ravine nature (fig. 2).

$$
\begin{aligned}
& f(x)=\sum_{i=1}^{n-1}\left(100 \cdot\left(x_{i+1}-x_{i}^{2}\right)^{2}+\left(x_{i}-1\right)^{2}\right), \\
& B=[-2.048,2.048]^{n} .
\end{aligned}
$$

Global minimum point and value: $x_{i}^{*}=1.0, f^{*}=0.0$.
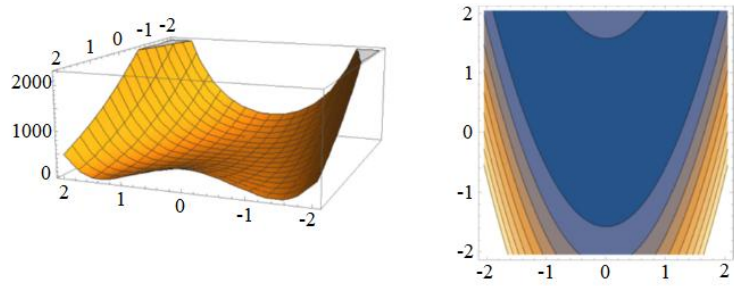

Fig. 2. Rosenbrock function plot (surface is on the left and level lines are on the right)

Table 2 shows the results of computations of finding a global minimum for each variant of the algorithm, where $N_{f}$ is the number of objective function evaluations.

TABLE II. RESULTS OF COMPUTATIONAL EXPERIMENTS FOR THE ROSENBROCK FUNCTION $(\mathrm{N}=10)$

\begin{tabular}{ccc}
\hline Variant & $\delta$ & $N_{f}$ \\
\hline $\mathbf{1}$ & $\mathbf{9 . 7 4 4 8 8 e - 0 7}$ & $\mathbf{1 3 2 5 4 6 3}$ \\
2 & $9.96842 \mathrm{e}-07$ & 3587698 \\
3 & $9.03972 \mathrm{e}-07$ & 2299844 \\
4 & $9.03972 \mathrm{e}-07$ & 2291307
\end{tabular}

Here, for example, 9.74488e-07 is the exponential form of the number $\delta$. The first version of the algorithm allowed to reach a global extremum with the least number of objective function evaluations.

\section{2) Rastrigin function}

The Rastrigin function proposed by L.A. Rastrigin in 1974 [9] is one of the classic non-convex optimization test problems [10-11]. It is multi-extremal, has several uniformly distributed local minima (fig. 3).

$$
\begin{aligned}
& f(x)=10 \cdot n+\sum_{i=1}^{n}\left(x_{i}^{2}-10 \cdot \cos \left(2 \pi x_{i}\right)\right), \\
& B=[-5.12,5.12]^{n} .
\end{aligned}
$$

Global minimum point and value: $x_{i}^{*}=0.0$, $f^{*}=0.0$.
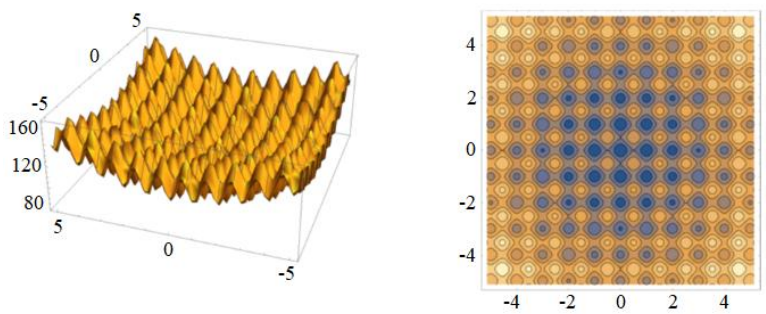

Fig. 3. Rastrigin function plot (surface is on the left and level lines are on the right) 
For the Rastrigin function, computational comparison of the variants of the multidimensional scheme was performed (see Table 3).

TABLE III. RESULTS OF COMPUTATIONAL EXPERIMENTS FOR THE RASTRIGIN FUNCTION $(N=10)$

\begin{tabular}{ccc}
\hline Variant & $\delta$ & $N_{f}$ \\
\hline 1 & $1.77636 \mathrm{e}-14$ & 1644 \\
$\mathbf{2}$ & $\mathbf{1 . 7 7 6 3 6 e - 1 4}$ & $\mathbf{1 6 0 3}$ \\
3 & $1.77636 \mathrm{e}-14$ & 7665 \\
4 & $1.77636 \mathrm{e}-14$ & 6763
\end{tabular}

The global extremum was reached with the help of all considered versions, the second version of the algorithm allowed us to get the result in fewer of the objective function calls.

\section{3) Ackley function}

The Ackley function is often used when testing global optimization methods $[10 ; 11]$. The problem is multi-extremal, it has a complex system of uniformly distributed local extremums (fig. 4).

$$
\begin{aligned}
& f(x)=20+e-20 \cdot e^{-0,2 \cdot \sqrt{\frac{1}{n} \cdot \sum_{i=1}^{n} x_{i}^{2}}}-e^{\frac{1}{n} \cdot \sum_{i=1}^{n} \cos \left(2 \pi x_{i}\right)}, \\
& B=[-30.0,30.0]^{n} .
\end{aligned}
$$

Global minimum point and value: $x_{i}^{*}=0.0$, $f^{*}=0.0$.
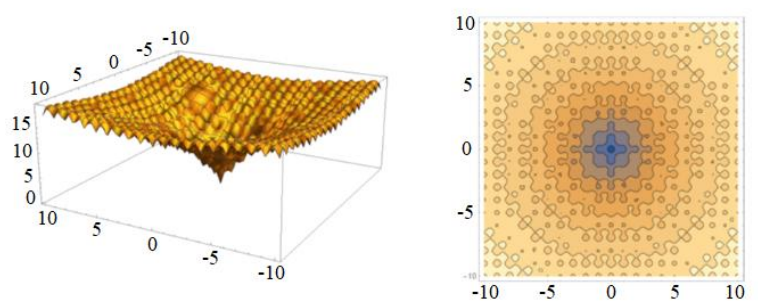

Fig. 4. Ackley function plot (surface is on the left and level lines are on the right)

Table 4 shows the results of computational experiments for the Ackley function.

TABLE IV. RESULTS OF COMPUTATIONAL EXPERIMENTS FOR THE ACKLEY FUNCTION $(N=10)$

\begin{tabular}{ccc}
\hline Variant & $\delta$ & $N_{f}$ \\
\hline 1 & $5.32287 \mathrm{e}-08$ & 1678 \\
$\mathbf{2}$ & $\mathbf{5 . 3 2 2 8 7 e - 0 8}$ & $\mathbf{1 5 0 6}$ \\
3 & $2.11111 \mathrm{e}-08$ & 8359 \\
4 & $2.11111 \mathrm{e}-08$ & 7143
\end{tabular}

Among the considered versions of the algorithm, the second version showed the best efficiency (using consistent fixation of the coordinates and the value of the parameter $K=50 \%$ ).
4) The model problem of optimal control for the power system

One of the traditional approaches to the study of the power system is a mathematical simulation by using controlled systems of differential equations. We present a well-known model energy problem considered in different sources [12], [13]. This system is a linearized model of the simplest power system: generator-tires of infinite power

$$
\begin{gathered}
\left\{\begin{array}{l}
\dot{x}_{1}=x_{2}(t) \\
\dot{x}_{2}=-6.725 x_{1}(t)-16.19 x_{3}(t)+0.19394 \\
\dot{x}_{3}=-0.066 x_{1}(t)-0.257 x_{3}(t)+0.19 u(t)+0.017566
\end{array}\right. \\
x_{1}(0)=0.115, x_{2}(0)=1.926, x_{3}(0)=0.055 \\
|u(t)| \leq 2.59, t \in[0,1.4], I(u)=\|x(1.4)\|^{2} \rightarrow \min .
\end{gathered}
$$

Here $u(t)$ is the excitation EMF, $x_{1}(t)$ is the rotor angle of the generator relative to the synchronous axis of a shift of the phase EMF and voltage. The problem considers the disturbing motion that occurs during accidents in power transmission. It is necessary in time $T=1.4$ to obtain a stable state of the system. In the process of minimization, the record value of the functional on the first stage was equal 0.00054 and obtained in a time of 1 CPU sec. The solution found on the second stage is 0.00127 by $593 \mathrm{sec}$ of CPU time and 569276 is the number of solved Cauchy problems.

The received results are presented in Fig.5 and Fig.6.

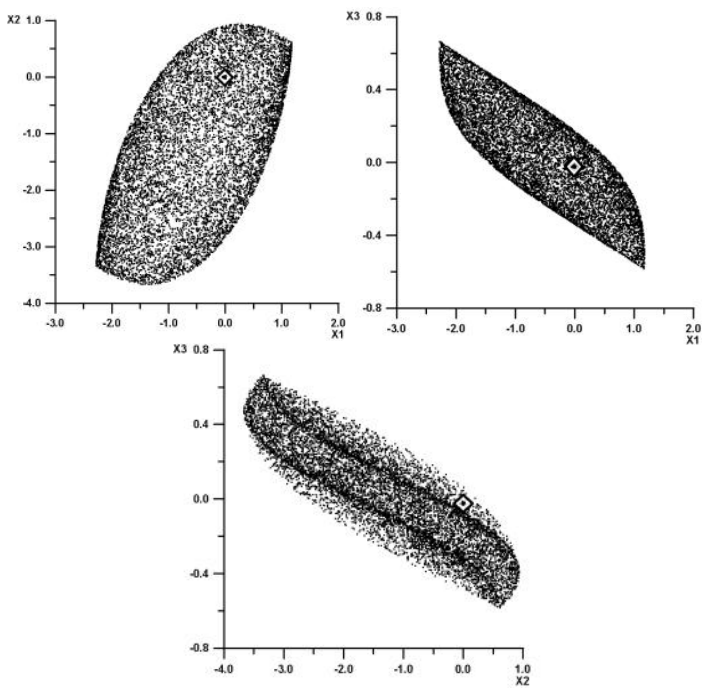

Fig. 5. The projections of reachable sets for the controlled power system. 


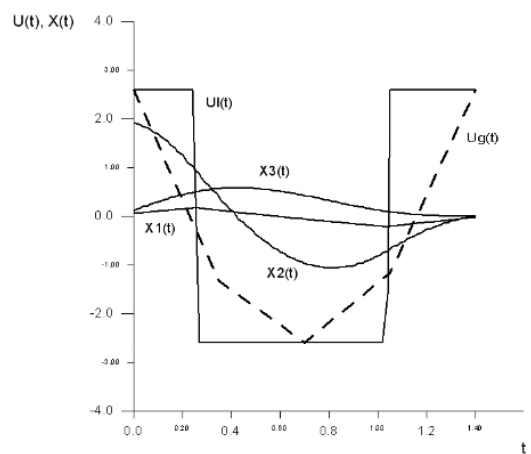

Fig. 6. Optimal trajectories and control (the dotted line for the first stage with five nodes and the solid line for the second stage).

\section{CONCLUSIONS}

The presented approach to the numerical study of the global extremum problems is based on the use of a parabolas algorithm and the methods of parabolic interpolation and the golden section for one-dimensional local search. It is carried out the testing of the implemented approach using well-known multiextremal functions. The proposed technique has been applied to investigate the model problem of optimal control for the power system. The obtained numerical results allowed us to demonstrate the efficiency of the proposed computational technology for global extremum search.

\section{ACKNOWLEDGEMENTS}

The work is partially supported by the Russian Foundation of Basic Research, grant No 17-07-00627.

\section{REFERENCES}

[1] Ya. D. Sergeev and D. E. Kvasov, Diagonal global optimization methods (Fizmatlit, Moscow, 2008). [Diagonal'nye metody global'noj optimizacii, Fizmatlit, Moskva, 2008] (In Russian)

[2] S. P. Shary, Finite interval interval analysis (Publishing house «XYZ», Novosibirsk, 2010). [Konechnomernyj interval'nyj analiz, Novosibirsk, 2010] (In Russian)
[3] A. Zhigljavsky and A. Zilinskas, Stochastic global optimization (Springer, New York, 2008).

[4] V. P. Gergel, V. A. Grishagin and A. V. Gergel, Multidimensional multiextremal optimization based on adaptive multi-step reduction of dimension, in Bulletin of the Nizhny Novgorod University named after N.I. Lobachevsky. 1 (2010) 163-170 [Mnogomernaya Mnogoekstremal'naya Optimizaciya Na Osnove Adaptivnoj Mnogoshagovoj Redukcii Razmernosti, Vestnik Nizhegorodskogo universiteta im. N.I. Lobachevskogo, 2010] (in Russian).

[5] V. P. Gergel, A Global Optimization for Multivariate Functions with Lipschitzian First Derivatives, in Journal of Global Optimization. 10(3) (1997) 257-281.

[6] T. S. Zarodnyuk and A. Yu. Gornov, Global extremum search technology in the optimal control problem, in Modern technologies. System analysis. Modeling. 3 (2008) 70-76 [Tekhnologiya poiska global'nogo ekstremuma $\mathrm{v}$ zadache optimal'nogo upravleniya, Sovremennye tekhnologii. sistemnyj analiz. modelirovanie, 3 (2008), 70-76] (in Russian).

[7] M. Jamil and X. S. Yang, A literature survey of benchmark functions for global optimisation problems, in International Journal of Mathematical Modelling and Numerical Optimisation. 4(2) (2013) 150-194.

[8] H. Rosenbrock, An automatic method for finding the greatest or least value of a function, in The Computer Journal. 3(3) (1960) 175-184.

[9] L. A. Rastrigin, Extreme control systems (Nauka, Moscow, 1974) [Sistemy ekstremal'nogo upravleniya, Nauka, Moskva, 1974] (in Russian).

[10] J. M. Dieterich and B. Hartke, Empirical review of standard benchmark functions using evolutionary global optimization, in Applied Mathematics. 3(10A) (2012) 1552-1564.

[11] K. Ding and Y. Tan, A CUDA-Based Real Parameter Optimization Benchmark, in Advances in Swarm Intelligence: 5th International Conference. Part II. (2014) 66-78.

[12] V. A. Baturin and D.E. Urbanovich, Approximate methods of optimal control, based on the principle of expansion (Nauka, Novosibirsk, 1997) [Priblizhennye metody optimal'nogo upravleniya, osnovannye na principe rasshireniya, Nauka, Novosibirsk, 1997](in Russian).

[13] A. I. Tyatyushkin, Numerical methods and software optimization of managed systems (Nauka, Novosibirsk, 1992) [CHislennye metody i programmnye sredstva optimizacii upravlyaemyh sistem, Nauka, Novosibirsk, 1992](in Russian). 